\title{
Avocado Extract Inhibits 7, 12-Dimethylbenz[a]anthracene (DMBA)- Induced Carcinogenesis in Hamster Cheek Pouches
}

\author{
Haiming Ding ${ }^{1 *}$, Bruce Casto ${ }^{2}$, Ye Deng ${ }^{3}$, Kevin Grill ${ }^{2}$, Wenrui Duan ${ }^{4}$, Ning Zhang ${ }^{5}$, Sara Cole ${ }^{6}$, Krista MD La Perle ${ }^{7}, \mathrm{Xueliang} \mathrm{Pan}^{8}$, A. \\ Douglas Kinghorn ${ }^{3}$ and Steven M. D'Ambrosio ${ }^{1}$ \\ ${ }^{1}$ Department of Radiology, College of Medicine, and Comprehensive Cancer Center, The Ohio State University, USA \\ ${ }^{2}$ College of Public Health, Division of Environmental Health Sciences, and Comprehensive Cancer Center, The Ohio State University, USA \\ ${ }^{3}$ Division of Medicinal Chemistry and Pharmacognosy, College of Pharmacy, The Ohio State University, USA \\ ${ }^{4}$ College of Medicine, Division of Medical Oncology, and Comprehensive Cancer Center, The Ohio State University, USA \\ ${ }^{5}$ Department of Neuroscience, College of Medicine, The Ohio State University, USA \\ ${ }^{6}$ Campus Microscopy \& Imaging Facility, The Ohio State University, USA \\ ${ }^{7}$ Department of Veterinary Biosciences, College of Veterinary Medicine, The Ohio State University, USA \\ ${ }^{8}$ Center for Biostatistics, The Ohio State University, USA
}

\begin{abstract}
Background/Aim: Our studies have shown that an avocado extract (D003) selectively inhibits proliferation in premalignant and malignant but not normal primary human oral epithelial cell lines via an ROS-mediated mechanism. Herein, the in vivo anticancer effect of D003 extract and freeze-dried avocado (FA) was determined in the DMBAinitiated hamster cheek pouch (HCP) model.
\end{abstract}

Materials and Methods: Tumors were initiated in hamster oral mucosa with DMBA followed by topical application of D003, FA, or vehicle. Tumor lesions were counted and evaluated histologically, epithelial proliferation was investigated using MTT assays, and ROS levels in HCP were examined using two photon microscopy.

Results: D003 significantly inhibited tumorigenesis (tumor number and volume) compared to FA and vehicle, but neither D003 nor FA reversed premalignant changes induced by DMBA. ROS levels that were increased in the mucosa by DMBA treatment were enhanced by D003. D003 significantly reduced proliferation of cells in DMBAinitiated mucosa.

Conclusion: Human cell culture and HCP data show that the phytochemicals extracted from avocado exhibit anticancer activity by inhibiting cancer cell proliferation and progression.

Keywords: Avocado extract; Cancer prevention; Cell proliferation; DMBA; Hamster cheek pouch

\section{Introduction}

Epidemiological investigations have suggested that the consumption of fruits and vegetables is associated with a lower risk of many different types of human cancer including oropharyngeal [1-6]. The protective effects of fruits and vegetables are thought to be related to their cancerpreventive components such as fiber, flavonoids, and polyphenols. As summarized in our previous review [7], avocado contains multiple phytochemicals with potential anticancer activity, among which are some aliphatic acetogenins that are structurally related to fatty acids, are rich and relatively special in avocado [8]. Ibiebele et al. [9] has reported that high intakes of n- 6 fatty acids from avocado, vegetables and nuts are inversely associated with risk of ovarian cancer, and Jackson et al. [10] have found that increased blood levels of oleic acid, another fatty acid, are associated with a reduced incidence of prostate cancer in Jamaica, where avocado is a major source of oleic acid in the diet.

Avocado extracts and isolated individual phytochemicals have shown anticancer activity in various types of human cancer cell lines such as oral, breast, and prostate $[7,8,11]$ in which extracts and individual phytochemicals may act by inhibiting cancer cell proliferation and inducing apoptosis. Our previously published studies have shown that aliphatic acetogenins, $(2 S, 4 S)$-2, 4-dihydroxyheptadec-16-enyl acetate and $(2 S, 4 S)-2,4$-dihydroxyheptadec-16-ynyl acetate, two components isolated from avocado, inhibit the EGRK/RAF/MEK/ERK1/2 oncogenic pathway [8]. Studies have also shown that avocado extracts up-regulate tumor suppressor genes such as p21 and p27, which are well-documented to block progression through the cell cycle, inducing $\mathrm{G}_{2} / \mathrm{M}$ cell-cycle arrest [12]
Reactive oxygen species (ROS) are a group of molecules and free radicals derived from molecular oxygen, mainly composed of superoxide, hydrogen peroxide, and their derivatives such as hydroxyl radical [13]. It is well documented that ROS play a multi-facet role depending on the type and concentration of ROS and the cell types [13]. On one hand, ROS are generated during the process of normal cellular metabolism and by enzymes in the mitochondria, endoplasmic reticulum, and cytosol. These ROS are important factors in signaling transduction involved in cell proliferation, differentiation, and cell death [13]. On the other hand, ROS may damage cellular DNA, which may lead to carcinogenesis [14,15]. Another well-documented phenomenon is that precancerous and cancerous cells contain an elevated level of endogenous ROS, and many cancer therapeutic agents eradiate these abnormal cells by further increasing the ROS [1619]. Similarly, our previous studies showed that D003, a chloroform extract of avocado, induced apoptosis in human oral premalignant and malignant epithelial cells, but not normal primary cells, via an ROSmediated mechanism [11]. These data suggest that an ROS mechanism may be involved in D003 inhibition of oral cancer cell proliferation and induction of apoptosis. In this study, 7,12-dimethylbenz [a] anthracene

*Corresponding author: Haiming Ding, Department of Radiology, College of Medicine, and Comprehensive Cancer Center, The Ohio State University, Room 730 BRT, 460 W 12th Avenue, Columbus, OH 43210, USA, Tel: 614-292-2453; Fax: 614-247-5050; E-mail: ding.29@osu.edu

Received May 28, 2014; Accepted July 01, 2014; Published July 03, 2014

Citation: Ding H, Casto B, Deng Y, Grill K, Duan W, et al. (2014) Avocado Extract Inhibits 7, 12-Dimethylbenz[a]anthracene (DMBA)-Induced Carcinogenesis in Hamster Cheek Pouches. Med chem S1: 008. doi:10.4172/2161-0444.S1-008

Copyright: (c) 2014 Ding H, et al. This is an open-access article distributed under the terms of the Creative Commons Attribution License, which permits unrestricted use, distribution, and reproduction in any medium, provided the original author and source are credited. 
(DMBA) treated hamster cheek pouches (HCP) were utilized to investigate the chemopreventive efficacy of concentrated, freeze-dried avocado and a chloroform extract, which may be more practical for use in humans.

\section{Materials and Methods}

\section{Agents}

FA from Hass avocado (Persea americana) was a kind gift from SIOSI (Mexico City, Mexico). Based on our studies in cell culture, both water and ethanol extracts from FA showed a similar potency (our unpublished data) to those of the fresh Hass avocado provided by the California Avocado Commission (CAC) that was used in our previous studies $[7,8,11]$. The FA contained the same two main active compounds previously isolated from Hass avocado fruit supplied by CAC, namely, $(2 S, 4 S)$-2,4-dihydroxyheptadec-16-enyl acetate and (2S,4S)-2,4-dihydroxyheptadec-16-ynyl acetate [8].

Avocado chloroform extract (D003) was prepared from FA as previously described [7,11]. Methylcellulose, 7,12-dimethylbenz[a] anthracene (DMBA), and N-acetyl-L-cysteine (NAC) were purchased from Sigma-Aldrich (Milwaukee, WI). DMBA was dissolved in dimethylsulfoxide (Fisher Scientific; Pittsburgh, PA) at a $0.2 \%$ concentration $(\mathrm{w} / \mathrm{v})$. FA (10\%) (w/v) and D003 extract $(2 \%)(\mathrm{w} / \mathrm{v})$ were mixed in $2 \%$ methylcellulose. The DMBA solution, FA and D003 extract mixtures were stored at $-20^{\circ} \mathrm{C}$, in $5 \mathrm{ml}$, aliquots and freshly thawed prior to each dosing. ROS/RNS detection kit, 4',6-diamidino-2phenylindole (DAPI), and OCT mounting medium were obtained from Enzo Life Sciences (Farmingdale, NY). MTT (3-(4,5-dimethylthiazol2-yl)-2,5-diphenyl-2 $H$-tetrazolium bromide) was purchased from Sigma-Aldrich (Milwaukee, WI). Isoflurane was obtained from Butler (Dublin, $\mathrm{OH}$ ).

\section{Animals}

Male Syrian golden hamsters (Mesocricetus auratus), 4-5 wks of age, were obtained from Charles River Laboratories (Wilmington, MA). The hamsters were housed in The Ohio State University AAALACapproved vivarium (three per cage) under standard conditions. Food AIN-76A (Dyets, Bethlehem, PA) and water were provided ad libitum. Hamsters were allowed to acclimate for $1 \mathrm{wk}$ before starting experiments. All experiments were approved by The Ohio State University Animal Care and Use Committee and conditions were in accordance with NIH Guidelines

\section{Experimental design}

Model for cancer prevention: To determine the cancer preventive effects of FA and D003 extract, hamsters were randomly divided into four groups $(\mathrm{N}=21)$ and both cheek pouches painted $3 \times /$ wk for 6 wks with $0.2 \%$ DMBA followed by treatment with chemopreventive agents $3 \times /$ wk for 6 wks. Group 1, 2\% methylcellulose; Group 2, 10\% FA; Group 3, 2\% D003 extract; and Group 4, untreated controls. The HCPs were treated topically with DMBA and chemopreventive agents using procedures described previously [20]. Briefly, hamsters were anesthetized by inhalation of a mixture of oxygen and 3-5\% isoflurane. The interior surface of the pouches was exposed using a metal retractor. For the first 6 wks, both HCPs in each hamster were painted three times weekly with $0.2 \%$ DMBA using a No. 4 sable hair brush using approximately $100 \mu \mathrm{L}$ for each application. For the second 6 wks, the control and avocado mixtures were applied three times weekly using a 16 g gavage needle attached to a $1 \mathrm{~mL}$ syringe $(200 \mu \mathrm{L}$ of either: $2 \%$ methylcellulose (Group 1), 10\% FA (Group 2), or 2\% D003 extract (Group 3). Food consumption was determined weekly and all animals were weighed biweekly. One wk after finishing the last application of the chemopreventive agents, animals were sacrificed by $\mathrm{CO}_{2}$ asphyxiation followed by cervical dislocation. Both cheek pouches were everted and gross tumors $\geq 1 \mathrm{~mm}^{3}$ were counted. Smaller leukoplakic lesions $(<1 \mathrm{~mm}$ in greatest diameter) were assessed by two different investigators by palpation of the pouch surface and visually at $2 \times$ magnification. Tumor size was determined with calipers and volume calculated using the formula: Volume $\left(\mathrm{mm}^{3}\right)=$ length $\times$ width ${ }^{2} \times 0.52$. The tumors were excised, divided into halves with $1 / 2$ placed in tissue cassettes for fixation in $10 \%$ neutral buffered formalin (NBF) at room temperature and $1 / 2$ frozen at $-70^{\circ} \mathrm{C}$. After $12 \mathrm{~h}$, formalin-fixed tumor tissues were transferred from NBF into phosphate buffered saline (PBS, $\mathrm{pH} 7.4$ ) and stored at $4^{\circ} \mathrm{C}$ pending paraffin embedding and tissue sectioning. The cheek pouches were also divided into halves and processed in the same manner.

Model for measuring ex vivo ROS in HCP and MTT assay: Based on our previous studies, HCPs show premalignant pathological changes after 3 wks of DMBA painting [20]. To investigate the change in ROS in premalignant mucosa, HCPs were treated with DMBA for 4 wks followed by D003 extract for 24 and $48 \mathrm{~h}$ using the same method as described above. Each treatment group contained $10 \mathrm{HCPs}$ from 5 hamsters. The HCPs were excised, washed twice in $5 \mathrm{~mL}$ PBS, soaked in $5 \mathrm{~mL}$ MEM with $10 \% \mathrm{FBS}$ and $100 \mathrm{U}$ penicillin/streptomycin, and then kept at $37^{\circ} \mathrm{C}$ supplemented with $5 \% \mathrm{CO}_{2}$. To ensure comparison of a similar location between pouches from different hamsters, the middle $1 / 3$ of each pouch (approximately $1.5 \mathrm{~cm}$ wide) was selected by placing the whole pouch over a glass test tube. The selected portions were then cut into $5 \times 5 \mathrm{~mm}^{2}$ pieces for two-photon microscopic examination and MTT assays.

Histology: Hamster pouches and tumors, that were fixed for $24 \mathrm{~h}$ in $10 \%$ neutral formalin and then transferred into PBS, were processed by routine methods through graded alcohols and xylenes, and embedded in paraffin. Sections $(4 \mu \mathrm{m})$ were stained with hematoxylin and eosin (HE) and evaluated with an Olympus BX45 light microscope with attached DP25 digital camera by a veterinary pathologist certified by the American College of Veterinary Pathologists (KMDL).

ROS detection: Fluorescence imaging and ROS detection were performed based on a published protocol [21]. One piece from each HCP was imaged using an Olympus FV100MPE microscope equipped with a 25XXL Plan, 1.05 N.A., and water immersion objective lens. Briefly, the tissues were placed on chamber slides, followed by addition of $100 \mu \mathrm{L}$ of MEM containing $15 \mu \mathrm{M}$ dihydrorhodamine 123 (DHR, an oxidative stress detection reagent) and DAPI $(0.5 \mu \mathrm{g} / \mathrm{ml})$ for $30 \mathrm{~min}$ at $37^{\circ} \mathrm{C}, 5 \% \mathrm{CO}_{2}$. The positive controls were $\mathrm{HCP}$ tissues of untreated control hamsters incubated in $100 \mu \mathrm{L}$ MEM containing $15 \mu \mathrm{M}$ DHR in the presence of $200 \mu \mathrm{M}$ pyocyanin (an ROS inducer) for $30 \mathrm{~min}$ at $37^{\circ} \mathrm{C}, 5 \% \mathrm{CO}_{2}$. The blocking controls were tissues from DMBA-treated pouches that were incubated with $15 \mu \mathrm{M}$ DHR in the presence of 5 $\mathrm{mM} \mathrm{N}$-acetyl-L-cysteine (an ROS inhibitor) at $37^{\circ} \mathrm{C}, 5 \% \mathrm{CO}_{2} .12$ bit images were captured with an excitation wavelength of $488 \mathrm{~nm}$. Images were captured using $2 \mu \mathrm{m}$ increments at various depths of the mucosa. Representative images were captured at the top of all samples. Average fluorescence intensity measurements of ROS-specific DHR signal in the original images (presented in Figure 1) was determined using Olympus Fluoview software (ver. 2.1c). All presented images (Figure 3) were processed identically using Fluoview software. Average fluorescence intensities from image stacks were determined from the original image series using the region measurements tool from Metamorph Premiere software (Ver. 7.7.6.0, Molecular Devices).

MTT assay: Freshly harvested HCPs were incubated in $500 \mu \mathrm{L}$ 
A

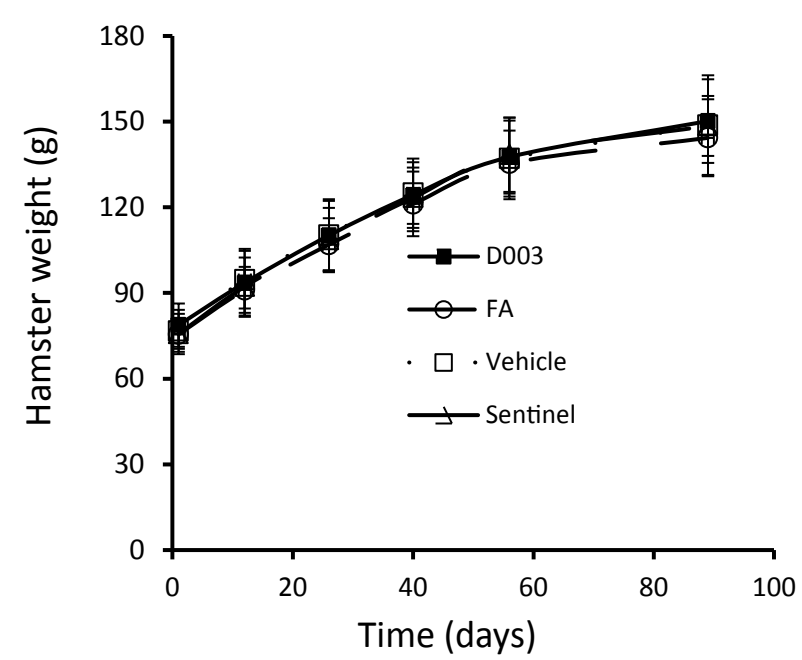

B

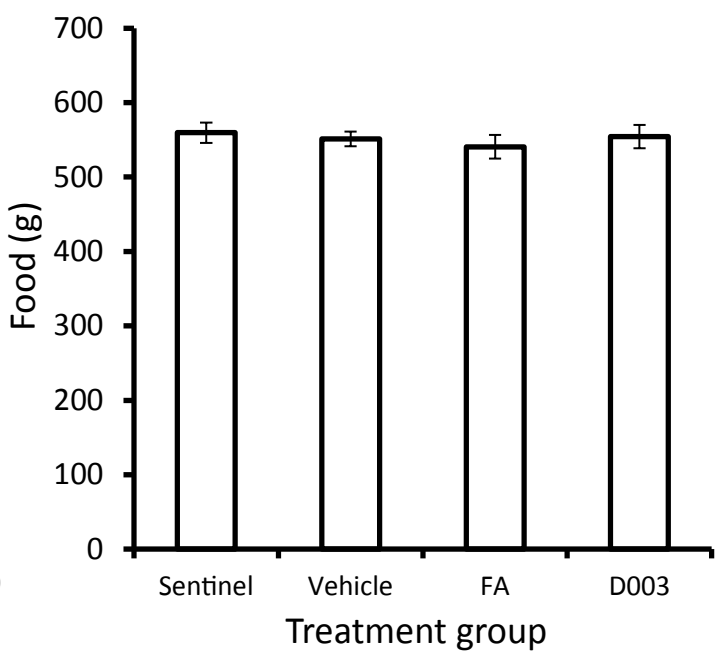

Figure 1: Application of avocado concentrated (FA) and extract (D003) do not significantly affect either weight gain ( $A$ ) or food consumption (B). Hamsters (21 in each group except sentinels which had 16) were treated with DMBA, FA, or D003 extract as described in Experimental Design. Food was measured and added once a wk while animals were weighed every other wk.

MEM with $10 \%$ FBS and $100 \mathrm{U}$ penicillin/streptomycin containing MTT $(0.5 \mathrm{mg} / \mathrm{ml})$ at $37^{\circ} \mathrm{C}, 5 \% \mathrm{CO}_{2}$ for $2 \mathrm{~h}$. The tissues were then embedded in OCT mounting media and stored at $-70^{\circ} \mathrm{C}$. The tissues were then cut into $10 \mu \mathrm{m}$ sections using a cryostat (Thermo, Michigan). The sections were then covered with mounting medium containing 0.2 $\mu \mathrm{g} / \mathrm{mL}$ DAPI to stain the nuclei of epithelial cells. The sections were viewed and images captured using an Olympus IX81 fluorescence microscope. The MTT formazan deposit in the epithelial cell layer was categorized based on the density of the color and size of the area as mild $(+)$, moderate $(+$ to ++$)$, or marked $(+++$ to ++++$)$.

\section{Statistical Analysis}

From the 42 pouches of the 21 hamsters in each group (control, FA and D003), tumors $\geq 1 \mathrm{~mm}^{3}$ were identified along with their volume. Results expressed as tumor incidence (i.e., percentage of pouches with tumors) and average tumor volume (the average volume of tumors per pouch, including only tumor-bearing pouches). The primary analysis compared tumor incidence and volume between the D003 and the control group. Tumor incidence between the two groups was compared using Fisher's exact test and the tumor volumes were compared using Mann-Whitney test. Secondary analyses included comparison of the FA with the control and the D003 treatment group in terms of tumor incidence and volume. Image data from MTT assay were analyzed using Wilcoxon Rank Sum Test to compare MTT deposit grades in HCPs of control, DMBA, and DMBA+D003. A $p$ value of 0.05 or less was considered significant.

\section{Results}

\section{Animal body weights and food consumption}

The potential effects of FA, D003 extract, and DMBA on the hamsters' general health were determined by animal appearance, food consumption, and interval weighing. As shown in Figure 1, there was no significant difference $(p>0.05)$ in weight gain or food consumption among the three groups compared to the untreated control group during the study period of 12 wks, indicating that neither FA nor D003 extract treatment had any adverse effect.

\section{Avocado and D003 extract inhibit DMBA-induced tumorigenesis in HCPs}

The effect of FA and D003 extract on DMBA-induced tumorigenesis in HCPs was analyzed after 6 wks of DMBA painting followed by 6 wks of topical treatment with vehicle, FA, or D003 extract. As shown in Figure 2, FA and D003 extract reduced both the number and size of DMBA-induced tumors in the HCPs. Specifically, Figure 2B shows that FA and D003 extract decreased the number of DMBA-induced tumors (19 in vehicle controls to 14 in FA $(p=0.36)$, and 9 in D003 $(p=0.036)$, respectively). Figure $2 \mathrm{C}$ shows that FA and D003 extract also significantly inhibited the total volume of DMBA-induced tumors from 1353.56 in vehicle controls to 1004.64 and $60.32 \mathrm{~mm}^{3}$, respectively. The average tumor size was decreased significantly by D003 from $71.2 \pm$ $122 \mathrm{~mm}^{3}$ in controls to $6.7 \pm 9.7 \mathrm{~mm}^{3}(p=0.046)$. Figure $2 \mathrm{D}$ shows that total lesions (including tumors $\geq 1 \mathrm{~mm}^{3}$ and small leukoplakic lesions) were inhibited. Histological evaluation of tissue sections from pouches treated with $\mathrm{DMBA}, \mathrm{DMBA}+\mathrm{FA}$, or DMBA+D003 showed dysplasia, hyperplasia/hyperkeratosis, papilloma, and papilloma-dysplasia. These data indicate that avocado contains active components that inhibit the progression of DMBA initiated dysplasia/hyperplasia to tumors.

\section{ROS change in ex vivo hamster cheek pouches}

To investigate whether D003 extract affects ROS levels in in vivo oral tissue, hamster pouches were painted with DMBA for 4 wks followed by D003 topical treatment for 24 or $48 \mathrm{~h}$. HCPs were harvested 24 or $48 \mathrm{~h}$ after application of D003 and were incubated in MEM containing DHR. Normal mucosa from the methyl cellulose treated control group and D003 treated control contained low background ROS signals at various depths of ex vivo mucosa tissue using fluorescence microscopy (average fluorescence intensity 96.9). Incubation of the control mucosa with an ROS inducer resulted in a marked increase in ROS signals over that of the negative control with average fluorescence intensity of 1240.9. Mucosa from DMBA-treated pouches harbored high ROS signals with an intensity similar to that of ROS-induced positive controls (average fluorescence intensity 805.9. The pouches treated with DMBA followed by application of D003 showed a similar ROS 

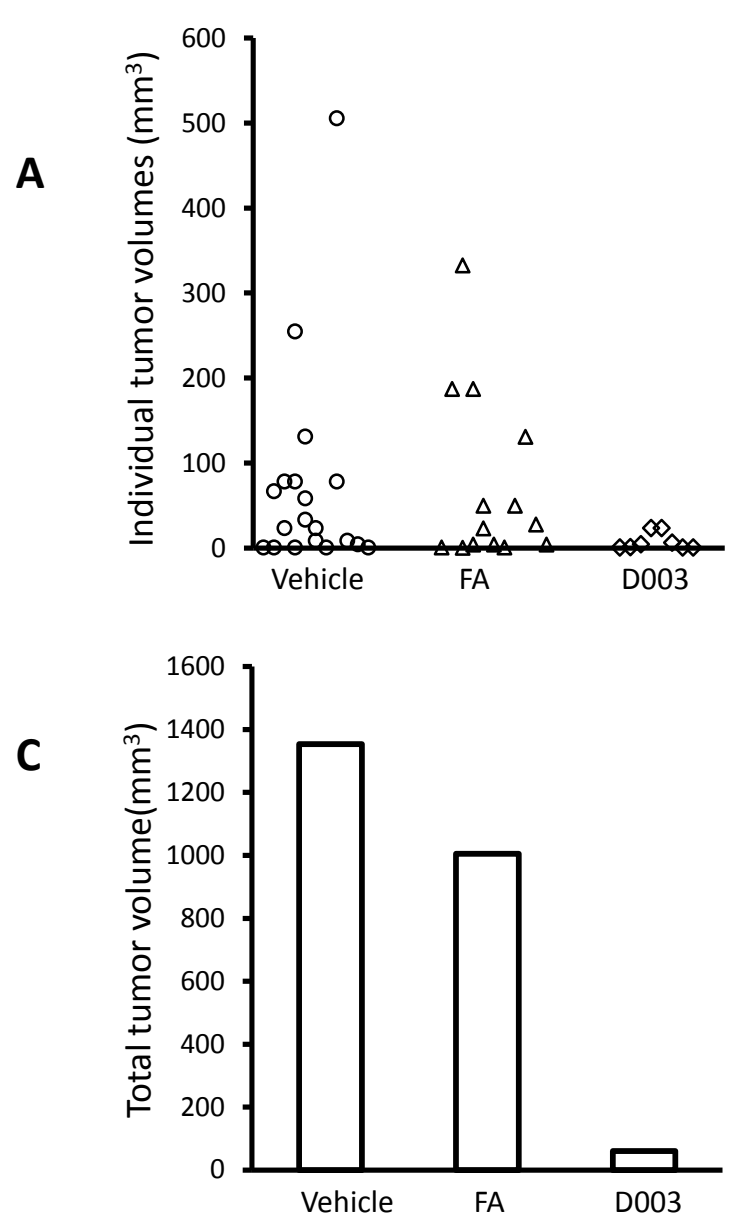
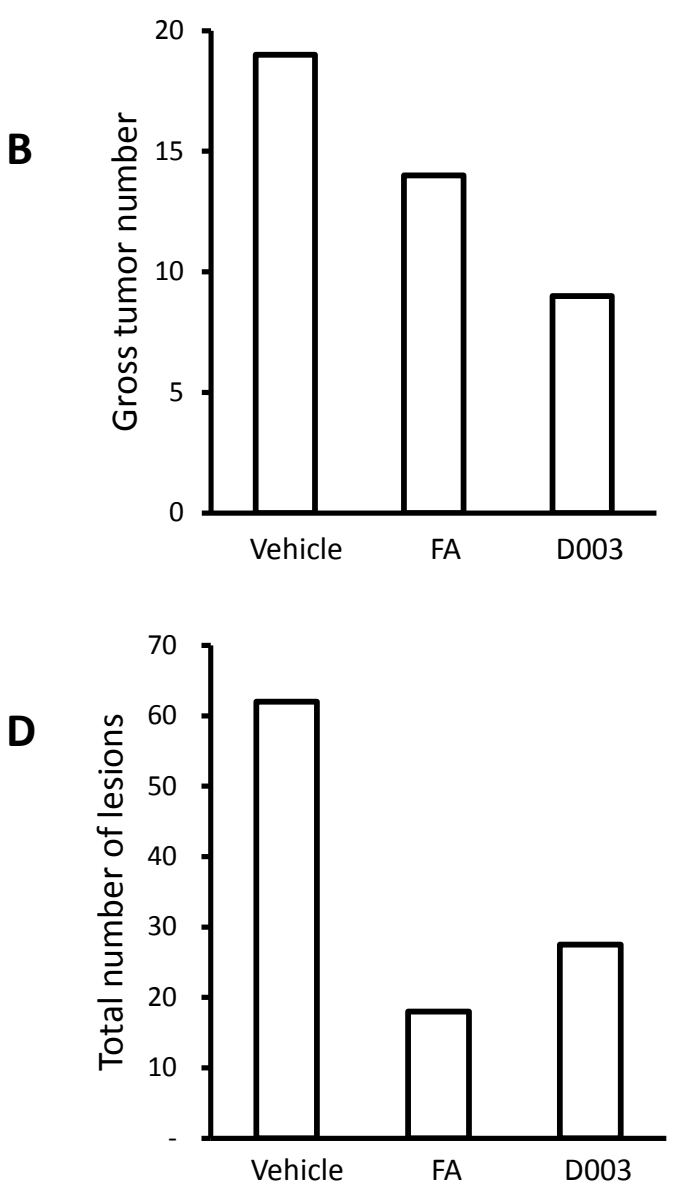

Figure 2: Application of concentrated avocado (FA) and extract (D003) inhibits DMBA-induced tumors in HCPs. Hamsters (21 in each group) were painted with DMBA for 6 wks followed by topical application of FA, D003 extract, or vehicle for 6 wks. Hamsters were sacrificed one wk after the last treatment and pouches were harvested and examined. (A) plot of all grossly visible tumors $\left(\geq 1 \mathrm{~mm}^{3}\right.$ ) (y axis: individual tumor volume; $x$ axis: each individual tumor); (B) total number of grossly visible tumors which were $\geq 1 \mathrm{~mm}^{3}$; (C) total volumes of grossly visible tumors which were $\geq 1 \mathrm{~mm}^{3} ;(\mathrm{D})$ total lesions including grossly visible tumors and leukoplakic lesions $<1 \mathrm{~mm}^{3}$.

signal as was observed with pyocyanin-induced positive controls (average fluorescence intensity 1422.7. The average fluorescence intensity of the ROS signals was determined from an image series through the thickness of the pouch mucosa. The average intensities ranged from 354.0 to 1405.4 in the induced positive control sample; 288.0 to 613.8 in the DMBA-initiated sample; and 301.3 to 2083.1 with DMBA treatment followed by application of D003. There were similar ROS signals in the DMBA-initiated pouches after treatment with D003 extract for 24 and $48 \mathrm{~h}$. Addition of NAC, an ROS scavenger, to the positive control group completely suppressed pyocyanin-induced ROS signal (data not shown). These data suggest that the D003 extract increases ROS levels in DMBA-initiated mucosa, which is consistent with the findings in our cell culture studies using premalignant human oral cell lines (Figure 3).

\section{Reduced proliferation by avocado extract in DMBA-initiated cheek pouches}

The metabolic and proliferative activity of viable HCP mucosa was determined by MTT assay. As shown in Figure 4, there was little (+) MTT deposition in normal control pouches from untreated animals (Figure 4A). Co-staining tissue with DAPI showed MTT located mainly in the basal layer. In contrast, there was a pronounced increase in MTT deposition $(+++$ to ++++$)$ in the pouches after 4 wks of treatment with
DMBA (Figure 4B). MTT was densely located in basal and superficial epithelial layers as well as the subjacent lamina propria. The DMBAtreated pouches when followed by application of the D003 extract for 24 (Figure 4C) and $48 \mathrm{~h}$ showed a markedly reduced MTT deposition $(+$ to ++$)$ in the pouches which was similar to the levels observed in the untreated controls. There are significant differences in MTT deposit grades between control, DMBA-treated HCPs, and DMBA + D003 $(p=0.007)$. This is also true between untreated controls and HCPs treated with DMBA (0.012), between DMBA and DMBA followed by D003 ( $p=0.037$ ). These data suggest that DMBA treatment significantly increases metabolic and proliferative activity in hamster HCP mucosa and that D003 extract reduces these activities.

\section{Discussion}

Studies by our laboratories and others have demonstrated that avocado contains anticancer phytochemicals [7,8]. Freeze-dried avocado, avocado chloroform extract, and two aliphatic acetogenins isolated from avocado selectively inhibit proliferation of premalignant and malignant human oral cancer cell lines [7,11]. Herein, we demonstrate that freeze-dried avocado (FA) and chloroform extract (D003) inhibit DMBA-induced oral tumors in hamster cheek pouches. To our knowledge, this is the first report that freeze-dried avocado and avocado extract inhibit tumors in the hamster oral model. 
Citation: Ding H, Casto B, Deng Y, Grill K, Duan W, et al. (2014) Avocado Extract Inhibits 7,12-Dimethylbenz[a]anthracene (DMBA)-Induced Carcinogenesis in Hamster Cheek Pouches. Med chem S1: 008. doi:10.4172/2161-0444.S1-008

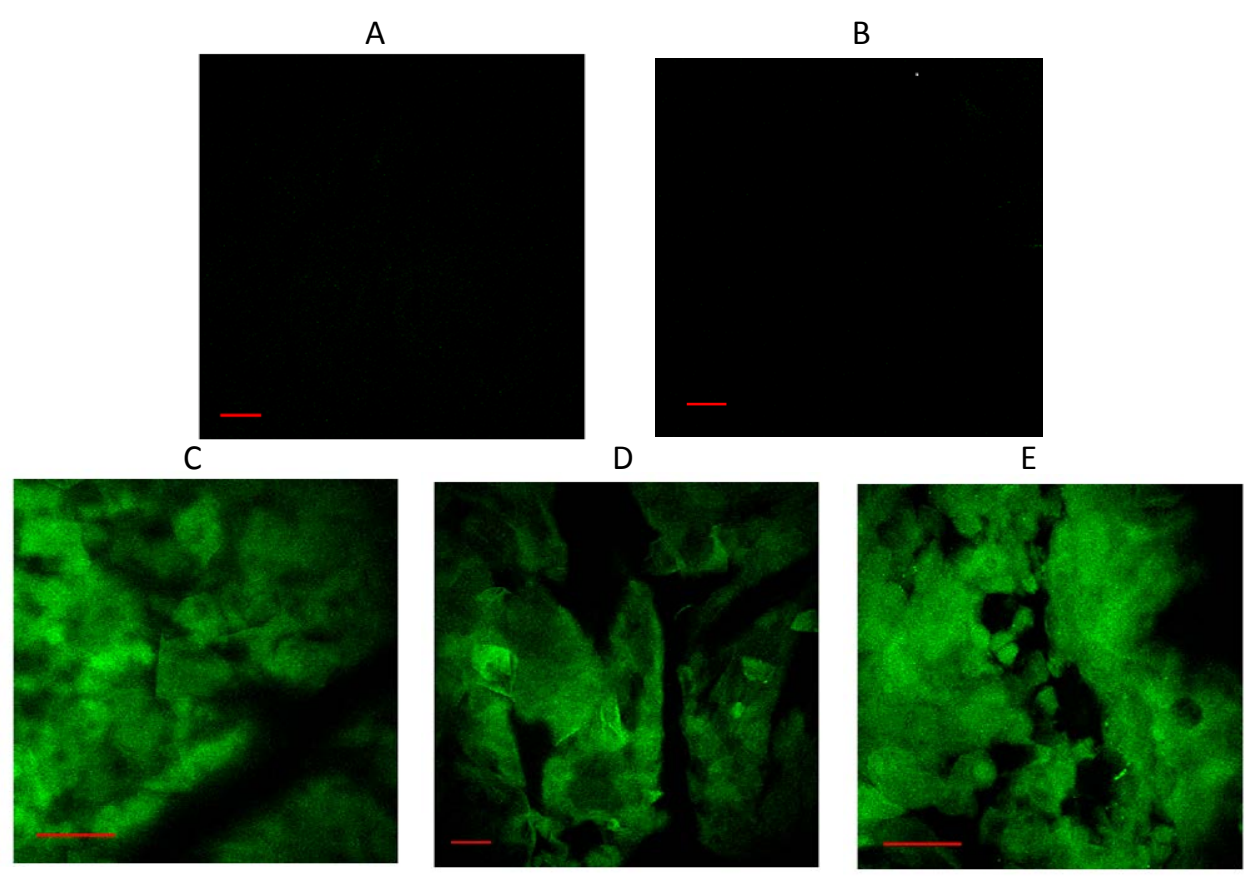

Figure 3: ROS changes in HCP mucosa. HCP pouches were excised from (A) sentinel control hamsters; (B) D003 control; (C) sentinel animal cheek pouches incubated with pyocyanin (an ROS inducer); (D) DMBA treatment for 4 wks followed by $2 \%$ methylcellulose for 24 h; (E) DMBA treatment for 4 wks followed by $2 \%$ D003 extract for $24 \mathrm{~h}$;. The mucosal samples were incubated in MEM containing $15 \mu \mathrm{M}$ DHR for 30 min and imaged with two-photon fluorescence microscopy at various depths. Photos taken at the top levels of all the tissues were shown as representative samples. Green signal indicates ROS and bars equal $50 \mu \mathrm{m}$.

A
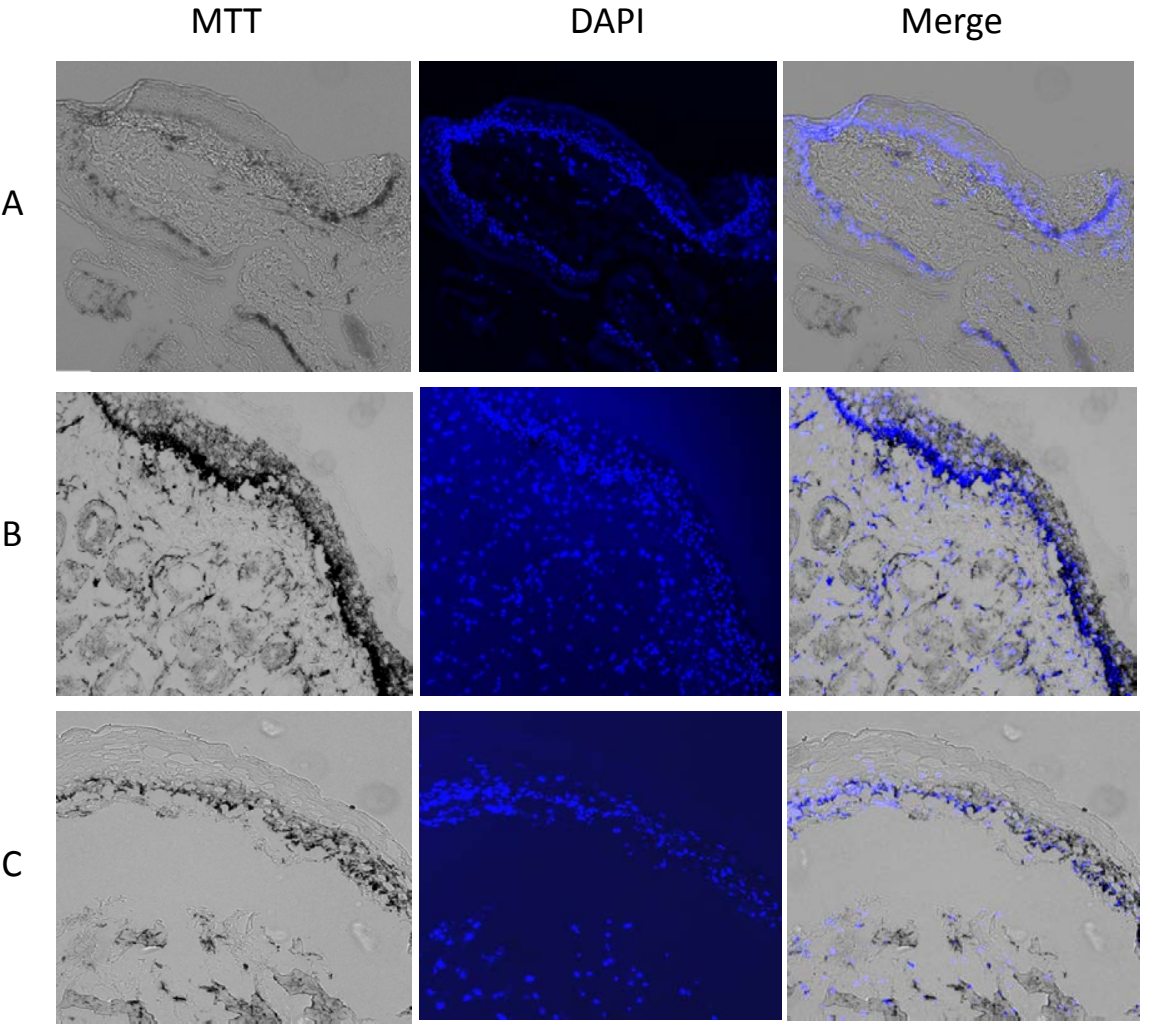

Figure 4: MTT deposition in DMBA-initiated HCP mucosa. HCPs were excised from (A) sentinel control hamsters; (B) DMBA (4 wks) followed by $2 \%$ methylcellulose $24 \mathrm{~h}$; (C) DMBA (4 wks) followed by $2 \%$ D003 extract $24 \mathrm{~h}$. The HCPs were incubated with MTT, processed into $10 \mu \mathrm{m}$ sections (left panel), and then co-stained with DAPI (middle panel) as described in Materials and Methods. MTT deposition shows as dark and black color (left and right panel). Nuclei were stained using DAPI and appear blue (middle and right panel). 
In the present study, we found that both FA and D003 extract inhibit DMBA-induced tumorigenesis. However, neither FA nor D003 extract markedly change the stage or frequency of existing dysplasia induced by DMBA in the HCP. This suggests that the cancer preventive effect of avocado extract is through inhibition of tumor progression, rather than reversing existing premalignant changes induced by carcinogen. This is consistent with the findings by Casto et al. [20,22] in cancer prevention studies with strawberry and black raspberry powders in which both were found to inhibit early lesions and tumor formation when agents were applied before and during carcinogen treatment, but did not affect either the stage or frequency of dysplastic lesions in preinitiated HCP. A slight decrease in histologic grade in 7/19 patients was reported by Shumway et al. [23] following topical application of black raspberry gel to dysplastic lesions in the human oral cavity. This discrepancy between human and hamster could be caused by variations in stages of the diseases or the different species that were used. Although both FA and D003 extract reduced the total number of lesions after long term treatment ( $6 \mathrm{wks}$ ), FA appeared to be more effective for reduction in total lesions while D003 was more effective in reducing the sizes of gross tumors. This differential effect might have been due to the difference in concentration or presence of multiple bioactive components (i.e., powder 10\% concentration vs. D003 extract $2 \%$ ). We assume that FA, as a whole concentrated food, contains more and different types of bioactive components and thereby may exert an effect on genes involved in both formation of dysplastic lesions and the conversion from hyperplasia to overt lesions. Alternatively, D003, containing higher concentrations of select chloroform soluble components, may inhibit progression from dysplastic lesions to gross tumors.

Human oral squamous cell carcinoma and the DMBA-induced HCP model both involve progression from epithelial hyperplasia into dysplasia and carcinoma. Human oral hyperplasia and dysplatia have been found to contain dysregulated gene expression such as loss of tumor suppressor gene p16, and overexpression of EGFR, Cyclin D1, and Ki-67 protein [24-28]. Buccal mucosa of hamsters treated with DMBA has demonstrated over-expression of cell proliferative markers PCNA and Cyclin D1 $[29,30]$. EGFR is known to activate downstream EGRK/RAF/MEK/ERK1/2 pathways [31], which further increase the expression of Cyclin D1 [32]. Cyclin D1 and p21 are key cell cycle genes controlling the cell cycle transition from $\mathrm{G}_{1}$ to $\mathrm{S}$, a major limiting phase for cell proliferation [33].

Clinical trials have shown that naturally-occurring agents have potential in treating precancerous lesions (leukoplakia/erthorplakia) [34-37]. Similar effects have been demonstrated in DMBA/HCP models $[20,22,38-44]$. This effect may be attributed to their ability to regulate multiple key cell cycle genes leading to inhibition of cell proliferation [45]. Garcinol from fruit rind of Garcinia spp., for example, has been found to inhibit cell proliferation in DMBAinduced HCP carcinogenesis [39]. Avocado extract has been shown to: i) inhibit EGRK/RAF/MEK/ERK1/2 pathways [8] and Cyclin D1 (our unpublished data) and over-expression of MEK/ERK1/2 that partially overcomes D003 reduced cell proliferation [8]; ii) up-regulate p21 [7] and our unpublished data); and, iii) enhance ROS and induction of apoptosis $[7,11,46]$. It has been reported that targeting EGFR in the DMBA/HCP model reduces cell proliferation [47]. In this study, using MTT, we showed that hyperplastic/dysplastic HCP tissues have a high cell proliferation rate that is inhibited by topical application of FA and D003 extract.

Enhancement of ROS has been identified in cell culture models as a critical mechanism by which many anticancer agents, including avocado extracts, damage cancer cells [16-19]. Therefore, high endogenous levels of ROS have been thought to be a double-edged sword to cancer development $[48,49]$. On one hand, high ROS damage DNA and stimulate cell proliferation; alternatively, ROS damage cancer cells when their level reaches a critical stage. Previous results have shown that early stage premalignant cell lines TE1177/HPV16/ E6 and TE1177/HPV16/E7 contain high levels of ROS; incubation with D003 extract increased their basal levels and induced apoptosis, but only caused a small increase in the primary parent TE1177 cells [11]. This was also found to be true in Jurkat lymphoblastic leukemia cells where avocado extract induced ROS-dependent apoptosis [46]. In the current study, we observed that DMBA treated buccal mucosa contained higher levels of ROS and that topical application of avocado D003 extract further increased the ROS levels. Whether increases in ROS in the mucosa of hamster cheek pouches helped to eliminate abnormal cells, as discovered in vitro needs further investigation.

In summary, these preliminary data demonstrated that freezedried avocado and its chloroform extract inhibited cell proliferation in buccal mucosa pre-initiated with DMBA and delayed/inhibited tumor development. Further evaluation of avocado extract for chemoprevention of human oral cancer is warranted.

\section{Acknowledgements}

We thank the NIDCR for grant support (R03DE020185 to HD), and SIOSI in Mexico for kindly providing freeze-dried avocado for our research.

\section{References}

1. Boffetta P, Couto E, Wichmann J, Ferrari P, Trichopoulos D, et al. (2010) Fruit and vegetable intake and overall cancer risk in the European Prospective Investigation into Cancer and Nutrition (EPIC). J Natl Cancer Inst 102: 529-537.

2. Cappellani A, Zanghì A, Di Vita M, Cavallaro A, Piccolo G, et al. (2013) Strong correlation between diet and development of colorectal cancer. Front Biosci (Landmark Ed) 18: 190-198.

3. Davidson PG, Touger-Decker R (2009) Chemopreventive role of fruits and vegetables in oropharyngeal cancer. Nutr Clin Pract 24: 250-260.

4. Gonzalez CA, Riboli E (2010) Diet and cancer prevention: Contributions from the European Prospective Investigation into Cancer and Nutrition (EPIC) study. Eur J Cancer 46: 2555-2562.

5. Key TJ (2011) Fruit and vegetables and cancer risk. Br J Cancer 104: 6-11.

6. Kim YS, Milner JA (2011) Bioactive food components and cancer-specific metabonomic profiles. J Biomed Biotechnol 2011: 721213.

7. Ding H, Chin YW, Kinghorn AD, D'Ambrosio SM (2007) Chemopreventive characteristics of avocado fruit. Semin Cancer Biol 17: 386-394.

8. D'Ambrosio SM, Han C, Pan L, Kinghorn AD, Ding H (2011) Aliphatic acetogenin constituents of avocado fruits inhibit human oral cancer cell proliferation by targeting the EGFR/RAS/RAF/MEK/ERK1/2 pathway. Biochem Biophys Res Commun 409: 465-469.

9. Ibiebele TI, Nagle CM, Bain CJ, Webb PM (2012) Intake of omega-3 and omega- 6 fatty acids and risk of ovarian cancer. Cancer Causes Control 23: 1775-1783.

10. Jackson MD, Walker SP, Simpson-Smith CM, Lindsay CM, Smith G, et al. (2012) Associations of whole-blood fatty acids and dietary intakes with prostate cancer in Jamaica. Cancer Causes Control 23: 23-33.

11. Ding H, Han C, Guo D, Chin YW, Ding Y, et al. (2009) Selective induction of apoptosis of human oral cancer cell lines by avocado extracts via a ROSmediated mechanism. Nutr Cancer 61: 348-356.

12. Butt AJ, Roberts CG, Seawright AA, Oelrichs PB, Macleod JK, et al. (2006) A novel plant toxin, persin, with in vivo activity in the mammary gland, induces Bim-dependent apoptosis in human breast cancer cells. Mol Cancer Ther 5 : 2300-2309.

13. Covarrubias L, Hernández-García D, Schnabel D, Salas-Vidal E, CastroObregón S (2008) Function of reactive oxygen species during anima development: passive or active? Dev Biol 320: 1-11. 
Citation: Ding H, Casto B, Deng Y, Grill K, Duan W, et al. (2014) Avocado Extract Inhibits 7,12-Dimethylbenz[a]anthracene (DMBA)-Induced Carcinogenesis in Hamster Cheek Pouches. Med chem S1: 008. doi:10.4172/2161-0444.S1-008

14. Indo HP, Davidson M, Yen HC, Suenaga S, Tomita K, et al. (2007) Evidence of ROS generation by mitochondria in cells with impaired electron transport chain and mitochondrial DNA damage. Mitochondrion 7: 106-118.

15. Pelicano H, Carney D, Huang P (2004) ROS stress in cancer cells and therapeutic implications. Drug Resist Updat 7: 97-110.

16. Evens AM, Lecane P, Magda D, Prachand S, Singhal S, et al. (2005) Motexafin gadolinium generates reactive oxygen species and induces apoptosis in sensitive and highly resistant multiple myeloma cells. Blood 105: 1265-1273.

17. Lee KW, Lee HJ (2006) The roles of polyphenols in cancer chemoprevention. Biofactors 26: 105-121.

18. Martin KR (2006) Targeting apoptosis with dietary bioactive agents. Exp Biol Med (Maywood) 231: 117-129.

19. Shakibaei M, Schulze-Tanzil G, Takada Y, Aggarwal BB (2005) Redox regulation of apoptosis by members of the TNF superfamily. Antioxid Redox Signal 7: 482-496.

20. Casto BC, Kresty LA, Kraly CL, Pearl DK, Knobloch TJ, et al. (2002) Chemoprevention of oral cancer by black raspberries. Anticancer Res 22: 4005-4015.

21. Lee EH, Faulhaber D, Hanson KM, Ding W, Peters S, et al. (2004) Dietary lutein reduces ultraviolet radiation-induced inflammation and immunosuppression. J Invest Dermatol 122: 510-517.

22. Casto BC, Knobloch TJ, Galioto RL, Yu Z, Accurso BT, et al. (2013) Chemoprevention of oral cancer by lyophilized strawberries. Anticancer Res 33: $4757-4766$.

23. Shumway BS, Kresty LA, Larsen PE, Zwick JC, Lu B, et al. (2008) Effects of a topically applied bioadhesive berry gel on loss of heterozygosity indices in premalignant oral lesions. Clin Cancer Res 14: 2421-2430.

24. Kövesi G, Szende B (2006) Prognostic value of cyclin D1, p27, and p63 in oral leukoplakia. J Oral Pathol Med 35: 274-277.

25. Nasser W, Flechtenmacher C, Holzinger D, Hofele C, Bosch FX (2011) Aberrant expression of $\mathrm{p} 53, \mathrm{p} 16 \mathrm{INK} 4 \mathrm{a}$ and $\mathrm{Ki}-67$ as basic biomarker for malignant progression of oral leukoplakias J Oral Pathol Med 40: 629-635.

26. Raju B, Mehrotra R, Oijordsbakken G, Al-Sharabi AK, Vasstrand EN, et al. (2005) Expression of p53, cyclin D1 and Ki-67 in pre-malignant and malignan oral lesions: association with clinicopathological parameters. Anticancer Res 25: 4699-4706.

27. Rousseau A, Lim MS, Lin Z, Jordan RC (2001) Frequent cyclin D1 gene amplification and protein overexpression in oral epithelial dysplasias. Oral Oncol 37: 268-275

28. Turatti E, da Costa Neves A, de Magalhães MH, de Sousa SO (2005) Assessment of c-Jun, c-Fos and cyclin D1 in premalignant and malignant oral lesions. J Oral Sci 47: 71-76.

29. Singh AK, Manoharan S, Vasudevan K, Rajasekaran D, Manimaran A, et al (2013) Anti-cell proliferative and anti-angiogenic potential of andrographolide during 7,12- dimethylbenz(a)anthracene induced hamster buccal pouch carcinogenesis. Asian Pac J Cancer Prev 14: 6001-6005.

30. Vidya Priyadarsini R, Kumar N, Khan I, Thiyagarajan P, Kondaiah P, et al. (2012) Gene expression signature of DMBA-induced hamster buccal pouch carcinomas: modulation by chlorophyllin and ellagic acid. PLoS One 7: e34628.

31. Seger R, Seger D, Reszka AA, Munar ES, Eldar-Finkelman H, et al. (1994) Overexpression of mitogen-activated protein kinase kinase (MAPKK) and its mutants in NIH 3T3 cells. Evidence that MAPKK involvement in cellular proliferation is regulated by phosphorylation of serine residues in its kinase subdomains VII and VIII. J Biol Chem 269: 25699-25709.

32. Terada Y, Nakashima O, Inoshita S, Kuwahara M, Sasaki S, et al. (1999) Mitogen-activated protein kinase cascade and transcription factors: the opposite role of MKK3/6-p38K and MKK1-MAPK. Nephrol Dial Transplant 14: $45-47$.
33. Sherr CJ, Roberts JM (1999) CDK inhibitors: positive and negative regulators of G1-phase progression. Genes Dev 13: 1501-1512.

34. Armstrong WB, Kennedy AR, Wan XS, Atiba J, McLaren CE, et al. (2000) Single-dose administration of Bowman-Birk inhibitor concentrate in patients with oral leukoplakia. Cancer Epidemiol Biomarkers Prev 9: 43-47.

35. Armstrong WB, Kennedy AR, Wan XS, Taylor TH, Nguyen QA, et al. (2000) Clinical modulation of oral leukoplakia and protease activity by Bowman-Birk inhibitor concentrate in a phase Ila chemoprevention trial. Clin Cancer Res 6: 4684-4691.

36. Cheng AL, Hsu CH, Lin JK, Hsu MM, Ho YF, et al. (2001) Phase I clinical trial of curcumin, a chemopreventive agent, in patients with high-risk or pre-malignant lesions. Anticancer Res 21: 2895-2900.

37. Tsao AS, Liu D, Martin J, Tang XM, Lee JJ, et al. (2009) Phase II randomized, placebo-controlled trial of green tea extract in patients with high-risk oral premalignant lesions. Cancer Prev Res (Phila) 2: 931-941.

38. Chandra Mohan KV, Hara Y, Abraham SK, Nagini S (2005) Comparative evaluation of the chemopreventive efficacy of green and black tea polyphenols in the hamster buccal pouch carcinogenesis model. Clin Biochem 38: 879-886.

39. Chen X, Zhang X, Lu Y, Shim JY, Sang S, et al. (2012) Chemoprevention of 7,12-dimethylbenz[a]anthracene (DMBA)-induced hamster cheek pouch carcinogenesis by a 5-lipoxygenase inhibitor, garcinol. Nutr Cancer 64: 12111218.

40. Garg R, Ingle A, Maru G (2008) Dietary turmeric modulates DMBA-induced p21ras, MAP kinases and AP-1/NF-kappaB pathway to alter cellular responses during hamster buccal pouch carcinogenesis. Toxicol Appl Pharmacol 232: 428-439.

41. Grawish ME (2008) Effects of Spirulina platensis extract on Syrian hamster cheek pouch mucosa painted with 7,12-dimethylbenz[a]anthracene. Oral Oncol 44: 956-962.

42. Kumar G, Tajpara P, Maru G (2012) Dietary turmeric post-treatment decreases DMBA-induced hamster buccal pouch tumor growth by altering cell proliferation and apoptosis-related markers. J Environ Pathol Toxicol Oncol 31: 295-312.

43. Letchoumy PV, Mohan KV, Prathiba D, Hara Y, Nagini S (2007) Comparative evaluation of antiproliferative, antiangiogenic and apoptosis inducing potentia of black tea polyphenols in the hamster buccal pouch carcinogenesis model. J Carcinog 6: 19.

44. Manoharan S, Balakrishnan S, Menon VP, Alias LM, Reena AR (2009) Chemopreventive efficacy of curcumin and piperine during 7,12-dimethylbenz[a] anthracene-induced hamster buccal pouch carcinogenesis. Singapore Med J 50: 139-146.

45. Gupta S, Ahmad N, Nieminen AL, Mukhtar H (2000) Growth inhibition, cellcycle dysregulation, and induction of apoptosis by green tea constituent (-)-epigallocatechin-3-gallate in androgen-sensitive and androgen-insensitive human prostate carcinoma cells. Toxicol Appl Pharmacol 164: 82-90.

46. Bonilla-Porras AR, Salazar-Ospina A, Jimenez-Del-Rio M, Pereañez-Jimenez A, Velez-Pardo C (2013) Pro-apoptotic effect of Persea americana var. Hass (avocado) on Jurkat lymphoblastic leukemia cells. Pharm Biol .

47. Sun Z, Sood S, Li N, Yang P, Newman RA, et al. (2008) Chemoprevention of 7,12-dimethylbenz[a]anthracene (DMBA)-induced oral carcinogenesis in hamster cheek pouch by topical application of a dual inhibitor of epidermal growth factor receptor (EGFR) and ErbB2 tyrosine kinases. Oral Oncol 44: 652 657

48. López-Lázaro M (2007) Dual role of hydrogen peroxide in cancer: possible relevance to cancer chemoprevention and therapy. Cancer Lett 252: 1-8.

49. Schumacker PT (2006) Reactive oxygen species in cancer cells: live by the sword, die by the sword. Cancer Cell 10: 175-176. 